Article

\title{
Generation of Complete SAR Geometric Distortion Maps Based on DEM and Neighbor Gradient Algorithm
}

\author{
Xiaohong Chen ${ }^{1}$, Qian Sun ${ }^{2,3, *}$ and Jun $\mathrm{Hu}^{1}{ }^{1}$ \\ 1 School of Geosciences and Info-Physics, Central South University, Changsha 410083, China; \\ chenxiaohong@csu.edu.cn (X.C.); csuhujun@csu.edu.cn (J.H.) \\ 2 College of Resources and Environmental Science, Hunan Normal University, Changsha 410081, China \\ 3 Key Laboratory of Geospatial Big Data Mining and Application, Hunan Province, Changsha 410081, China \\ * Correspondence: sunqian200241@aliyun.com; Tel.: +86-151-1110-0330
}

Received: 29 September 2018; Accepted: 7 November 2018; Published: 9 November 2018

Featured Application: The work can exploit open-source digital elevation model (DEM) to investigate the applicability of the available synthetic aperture radar (SAR) data in mountainous areas and is therefore helpful in the selecting or optimizing of the best collocate of SAR data in the monitoring of landslides.

\begin{abstract}
Radar-specific imaging geometric distortions (including foreshortening, layover, and shadow) that occur in synthetic aperture radar (SAR) images acquired over mountainous areas have a negative impact on the suitability of the interferometric SAR (InSAR) technique to monitor landslides. To address this issue, many distortion simulation methods have been presented to predict the areas in which distortions will occur before processing the SAR image. However, the layover and shadow regions are constituted by active as well as passive subregions. Since passive distortions are caused by active distortions and can occur in the flat area, it is difficult to distinguish the transition zone between passive distortion and non-distortion areas. In addition, passive distortion could cover part of the foreshortening or active layover/shadow areas but has generally been ignored. Therefore, failure to simulate passive distortion leads to incomplete simulated distortions. In this paper, an algorithm to define complete SAR geometric distortions and correct the boundaries among different distortions is presented based on the neighbor gradient between the passive and active distortions. It is an image-processing routine applied to a digital elevation model (DEM) of the terrain to be imaged by the available SAR data. The performance of the proposed method has been validated by the ascending and descending Advanced Land Observing Satellite (ALOS) Phased Array type L-band Synthetic Aperture Radar (PALSAR) images acquired over the Chongqing mountainous area of China. Through the investigation of passive distortion, we can have a deeper understanding of the formation and characteristics of these distortions. Moreover, it provides very meaningful information for research on areas such as landslide monitoring.
\end{abstract}

Keywords: InSAR; DEM; passive distortion; active distortion; neighbor gradient; landslides

\section{Introduction}

It is acknowledged that geometric distortion is an inherent error of synthetic aperture radar (SAR) images because of side-looking geometry and topographic relief. SAR geometric distortion (SGD) can be divided into various types, i.e., foreshortening, layover, and shadow. Furthermore, in the object space, the layover and shadow regions can be divided into active and passive subregions [1]. 
The presence of SGD has many negative effects on SAR images, especially those acquired over the mountainous areas. For instance, the SAR signal is useless in areas affected by layover and shadow [2], which makes phase unwrapping immensely difficult. This is the main reason the application of interferometric SAR (InSAR) techniques has been hampered in mountainous areas [3,4]. In addition, those distortions are bound to result in imprecise classifications of land use [5], inconsistencies in the digital elevation model (DEM) [6,7], and underestimation for deformation areas [8]. Since the SGD is the inherent error of the SAR image, many researchers prefer distortion simulation to distortion correction. This is of great importance since with simulated distortions, we can calculate the best imaging geometry without the SAR image, which provides us with a chance to select the most suitable SAR data for the interested area.

During the last two decades, several approaches have been developed to simulate the SGD. Kropatsch and Strobl [1] proposed a layover and shadow map (LSM) algorithm to simulate the layover and shadow, including active as well as passive ones. However, many parameters, such as height, imaging time measured in arc length along the flight path, and nadir distance, are required in the method, leading to many uncertainties in the generation of the layover/shadow. Rees [9] presented a simple algorithm based on the surface slope that can be applied very rapidly to indicate those areas only corresponding to active shadow and layover. Notti and colleagues $[10,11]$ integrated the synthesized effects of local topography into the R-index algorithm. Although the R-index can successfully identify the areas of foreshortening, it only accounts for the effects of active layover and cannot identify areas of passive layover and shadow (either active or passive). Plank et al. [12] presented a GIS-based imitated InSAR procedure by using the "one-observer" method to predict areas where layover and shadow will occur. However, this method requires a very large DEM that would reduce the accuracy of the layover-shadow simulation. Cigna et al. [13] combined the LSM and R-index algorithms to generate foreshortening, layover (both active and passive), and shadow (both active and passive). However, this method does not correct the boundaries among the distortions as a result of ignoring the spatial relationship with passive distortion, which will yield inaccurate simulation results.

In 2006, Colesanti and Wasowski [14] proposed a method for simulating the foreshortening and active layover/shadow (hereafter referred to as F-ALS). The F-ALS method identifies the ranges of aspect and slope which determine the foreshortening and active layover/shadow by analyzing the visibility conditions of unstable hillsides with different orientations [15]. Nevertheless, there is an obvious limitation to the F-ALS method in that it is incapable of deriving passive distortion, since the transition zone between passive distortion and non-distortion cannot be confirmed. This leads to incorrect coverage of the simulated distortion and makes it difficult to predict the distribution of persistent scatters. It is worth noting that passive layover can be employed to calculate the height of a building by creating a template based on the building's outline [16]. Looting can also be investigated by exploiting the relationship between the range of the passive layover and shadow. However, the relationship cannot be exhaustive of all possible combinations that can be found in real-world situations [17]. Therefore, the investigation of passive distortion is conducive to deepening the understanding of the terrain structure in geometric distortion areas.

Based on the F-ALS method, in this paper, we propose an innovative algorithm, featuring the passive distortion simulation, to generate the complete SGD maps from the DEM. This algorithm exploits the neighbor gradient between passive and active distortion, and therefore can identity the passive distortion in addition to the active one (hereafter referred to as P-NG). Furthermore, we reveal the interactions among layover, shadow, and foreshortening, which is the first attempt to do so to our knowledge. The boundaries among the different SGD effects are corrected by the P-NG method to improve the accuracy of the simulated results. 


\section{Methodology}

\subsection{Overview of the SGD}

For the slopes facing the SAR sensor, when the local terrain slope angle is smaller than the local incidence angle, such slopes have a shorter length in the SAR image than the flat terrain, which means the range resolution on such slopes becomes worse. This is the so-called foreshortening effect, which achieves the worst performance when the local terrain slope angle equals the local incidence angle (Figure 1a). Furthermore, when the local terrain slope angle exceeds the local incidence angle, the bottom and the top of such slopes are reverse imaged, which is known as the active layover effect. For the slopes facing away from the SAR sensor, when the local terrain slope angle is smaller than the complementary angle of the local incidence angle, the range resolution on such slopes becomes better in the SAR images than the flat terrain. Obviously, the resolution-enhancing effect is popular in InSAR measurements. However, when the local terrain slope angle exceeds the complementary angle of the local incidence angle, such steep slopes are completely prevented from receiving the SAR signal by the mountain itself. This is the active shadow effect that makes the slopes dark in the SAR image. For more details on geometric distortion analysis, see [14,15]. In addition, in the object space, the layover region splits into active and passive subregions. Active layover regions are the sources for layover, whereas passive layover regions are induced by the actives lying over them (Figure 1b). The active layover region is embedded in two passive regions called "near passive" and "far passive". The near passive region is closer to the sensor than the far passive region. Generally, the layover regions are much brighter than other places on the SAR image. In contrast to the layover, where two passive regions occur, here we have only one passive region for the shadow, called the "passive shadow" region. It is located at the end of the active shadow, where it cannot be reached by the radar beam since it is blanked by the active shadow regions (Figure 1c).
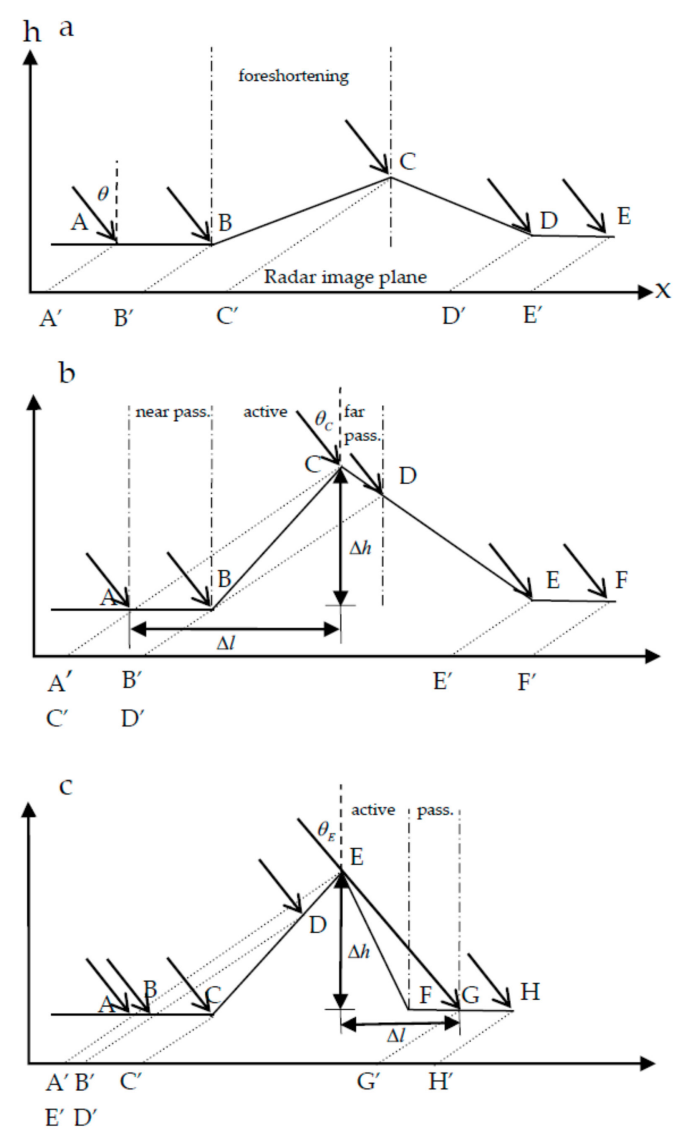

Figure 1. Illustrations of foreshortening (a), layover (b), and shadow (c). 


\subsection{F-ALS Method and Its Limitations}

The SGDs to be considered are those in the line of sight (LOS) of the SAR image, which can be mapped onto the ground plane of the DEM. For simplicity, we assume a locally flat earth. This is a rough approximation for calculating the local terrain slope and incidence angle, of which the results are not distorted by this assumption. Figure 1 illustrates the phenomena of foreshortening, layover, and shadow in the object space (DEM) and are detailed below.

Figure 1a shows scatterers A-E on the surface of a hill illuminated by the radar. The points $\mathrm{A}^{\prime}-\mathrm{E}^{\prime}$, located on the radar image plane, represent the positions where the scatterers A-E are imaged. The lengths of $\mathrm{A}^{\prime} \mathrm{B}^{\prime}$ and $\mathrm{D}^{\prime} \mathrm{E}^{\prime}$ equal those of $\mathrm{AB}$ and $\mathrm{DE}$, respectively, while the length of $\mathrm{B}^{\prime} \mathrm{C}^{\prime}$ is shorter than that of $\mathrm{BC}$. This means that the image is geometrically distorted in the sense that the hill appears to tilt towards the radar. This is the phenomenon of foreshortening.

Figure $1 \mathrm{~b}$ shows a steep hill. It is seen that the slope $B C$ is imaged in reversed order and is superimposed on the images of the sections $A B$ and $C D$. This is the called layover, where the slope $B C$ is also referred to as the "active layover", whereas sections $A B$ and $C D$, located at the both ends of the slope BC, are "near passive" and "far passive", respectively. Figure 1c shows an even steeper hill. In this case, the section of terrain EFG cannot receive the signal transmitted by the radar, leading to points E-G not being imaged. This is the phenomenon of shadow, where the slope EF is defined as the "active shadow", whereas the section FG, located at the end of the slope EF and which cannot be reached by the radar beam since it is blanked by the hillside EF, is referred to as the "passive shadow".

Except for passive distortion, these phenomena are related to the surface slope angle $\chi$ and incidence angle $\theta$, which can be calculated by the F-ALS method based on the relationship between the slope angle acquired by DEM and the incidence angle acquired by SAR imaging geometry:

$$
\begin{aligned}
& \text { facing-sensor }\left\{\begin{array}{l}
\chi_{i j}<\theta_{i j} \text { foreshortening } \\
\chi_{i j}=\theta_{i j} \text { worst - resolution } \\
\chi_{i j}>\theta_{i j} \text { active - layover }
\end{array}\right. \\
& \text { facing-away-sensor }\left\{\begin{array}{l}
\chi_{i j}<90-\theta_{i j} \text { resolution-enhancing } \\
\chi_{i j}>90-\theta_{i j} \text { active - shadow }
\end{array}\right.
\end{aligned}
$$

where the DEM grid is defined by $(i, j)$ with $i=1, \ldots, n ; j=1, \ldots, m, \chi_{i j}$ is the slope angle of each point of the DEM, and $\theta_{i j}$ is the incidence angle of each point of the SAR.

This indicates that the areas affected by the foreshortening and active layover/shadow can be identified by the F-ALS method but cannot be when identifying the areas affected by the passive distortion. Therefore, it is important to develop a method that can simulate passive distortion.

\subsection{P-NG Method for Simulating Passive Distortion}

The P-NG method defines for each cell whether it belongs to a passive layover region, a passive shadow region, or neither. Therefore, it is represented in the DEM geometry, which is generally a map projection. In the following algorithm, the values of the discrete points are determined on a pixel-by-pixel basis.

In this subsection, we focus on the P-NG method for defining the passive distortion. For obtaining the passive distortion, we need to determine the neighbor gradient between the active and passive distortions. In this paper, the mentioned neighbor gradient refers to the ratio of the height difference and ground distance between the two ground points, which can be approximated by

$$
N G=\frac{\Delta h_{i j, i k}}{\Delta l_{i j, i k}}
$$

where

$$
\Delta h_{i j, i k}=\left|h_{i j}-h_{i k}\right|
$$




$$
\Delta l_{i j, i k}=g r \cdot|j-k|
$$

where $\theta_{i j}$ and $h_{i j}$ are the incidence angle and elevation value of the climacteric point $(i, j)$ of the active distortion, respectively, $\Delta h_{i j, i k}$ is the height difference between the climacteric point $(i, j)$ of the active distortion and the point $(i, k), \Delta l_{i j, i k}$ is the ground range from the point $(i, k)$ to the climacteric point of the active distortion, and $g r$ is the ground range resolution of the DEM.

The point $(i, k)$ can be defined as the passive layover distortion when

$$
N G>\tan \theta_{i j}
$$

The point $(i, k)$ can be defined as the passive shadow distortion when

$$
\frac{1}{N G}<\tan \theta_{i j}
$$

\section{- $\quad$ Algorithm P-NG Generation:}

Phase 1: For each point $p_{i j}$, incidence angle $\theta_{i j}$ and slope angle $\chi_{i j}$ are calculated by using the F-ALS method. For active layover, if $\chi_{i j}>\theta_{i j}$ and towards the sensor (whereas, for active shadow, if $\chi_{i j}>90-\theta_{i j}$ and away from the sensor), which are the conditions for an active distortion pixel, $p_{i j}$ is marked as being active distortion. After phase 1, the P-NG contains all active distortion pixels. It is the input for phase 2 where the areas of the passive distortion are searched.

Phase 2: Column searching the passive distortion pixels for passive layover with Equation (5), and for passive shadow, with Equation (6). The searching starts with the climacteric point of an active distortion region. When the pixel is not satisfied with Equations (5) or (6), it acts as the boundary point of the passive distortion region and we search for the next passive distortion region. The intermediate pixels on the way from an active boundary to the corresponding passive boundary yield the passive distortion pixels.

Algorithm P-NG Generation is a point operation. Phase 2 should be done in parallel for every column and for every active distortion on that column.

During the procedure, it is necessary to pay attention to the selection of the search area starting from the climacteric point that is separated from the passive region. This greatly reduces the time burden of the calculation. In addition, for the passive layover, it should be calculated in two parts for the region, which is composed of the near and far passives.

\subsection{Interaction of the $S G D$}

For detailed studies of all interactions among the distortions, only five different cases of the interactions are possible:

- (D1) Interaction of passive layover and foreshortening

- (D2) Interaction of passive layover and active shadow

- (D3) Interaction of passive layover and passive shadow

- (D4) Interaction of passive shadow and foreshortening

- (D5) Interaction of passive shadow and active layover

They are shown in Figure 2 and can be derived from Equations (1)-(6).

The correction method for the boundaries of the SGD is based on the imaging sequence, mainly including: (A) For cases (D1) and (D2), the superposition is the passive layover, and the regions of foreshortening and active shadow can be corrected through Equations (1) and (5); (B) In the presence of cases (D2) and (D3) at the same time, the superposition is also defined as passive layover, whereas the un-overlapped passive shadow should be categorized as foreshortening or non-distortion areas; (C) For case (D3), the superimposed part is passive shadow; (D) In the cases of (D4) and (D5), the superimposed part is passive shadow, which can be corrected through Equations (1) and (6); (E) In the presence of 
(D3) and (D5) simultaneously, the superposition is passive shadow, while the un-overlapped passive layover should be classified as foreshortening or non-distortion areas.

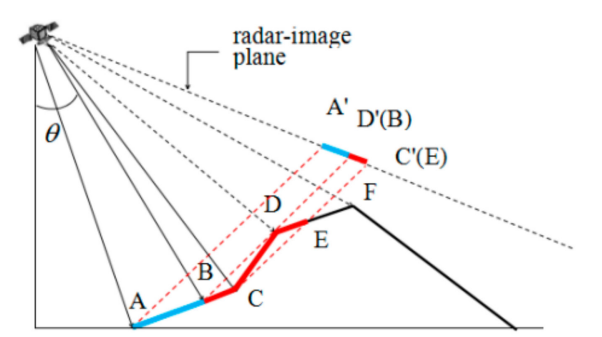

a. Interaction of passive layover with foreshortening

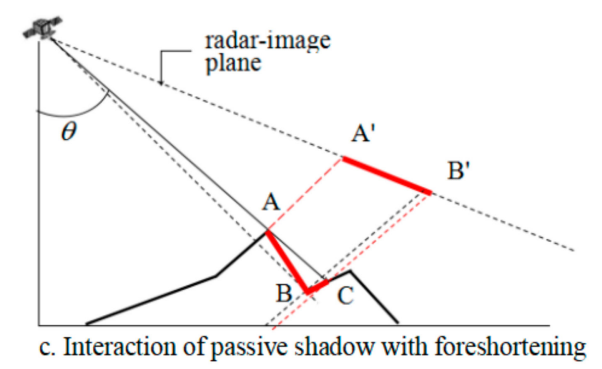

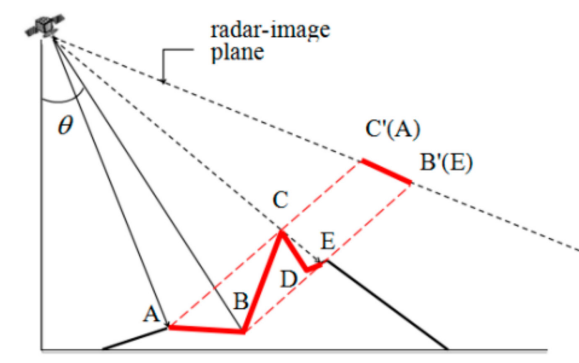

b. Interaction of passive layover with shadow

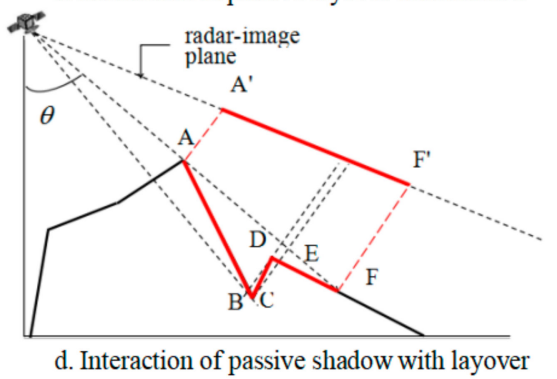

Figure 2. Interactions of the SGD.

Through studying the spatial relationship among the SGD, we have a deeper understanding of the formation and characteristics of the SGD. Additionally, the active distortion does not interact with foreshortening or itself. Conversely, the most of overlapping areas are the passive distortion with other distortions or itself, including the active distortion and the foreshortening. The P-NG method presented in this paper cannot only obtain the passive distortion on a pixel-by-pixel basis in the light of the neighbor gradient between active and passive distortion but can also correct the boundaries among the distortions to yield accurate simulation areas.

\section{Experiments and Results}

\subsection{Study Area and Used Data}

As shown in Figure 3, the study area is in Chongqing, southwest of Sichuan, China [18-20]. Influenced by the subtropical humid monsoon climate, rainfall is abundant, which has triggered thousands of landslides. However, the landslides are generally located in a mountainous area, which is difficult to monitor by the InSAR technique. Therefore, it is essential to analyze the SGD mechanisms in the study area to select the optimal SAR pairs.

To test the proposed P-NG method, 1 arc-sec Shuttle Radar Topography Mission (SRTM) DEM data was acquired for the study area. In addition, ascending and descending Advanced Land Observing Satellite (ALOS) Phased Array type L-band Synthetic Aperture Radar (PALSAR) image pairs were selected to provide the actual SGD map used for comparison (see Table 1).

Table 1. Parameters of the formed ALOS PALSAR image pairs. B_para and B_temp represent the spatial baseline and time interval between the two acquisitions, respectively.

\begin{tabular}{cccccc}
\hline No. & Orbit & Master & Slave & B_Temp (day) & B_Para (m) \\
\hline 1 & Ascending & 20081201 & 20090116 & 46 & 290 \\
2 & Descending & 20080908 & 20081024 & 46 & 146 \\
\hline
\end{tabular}




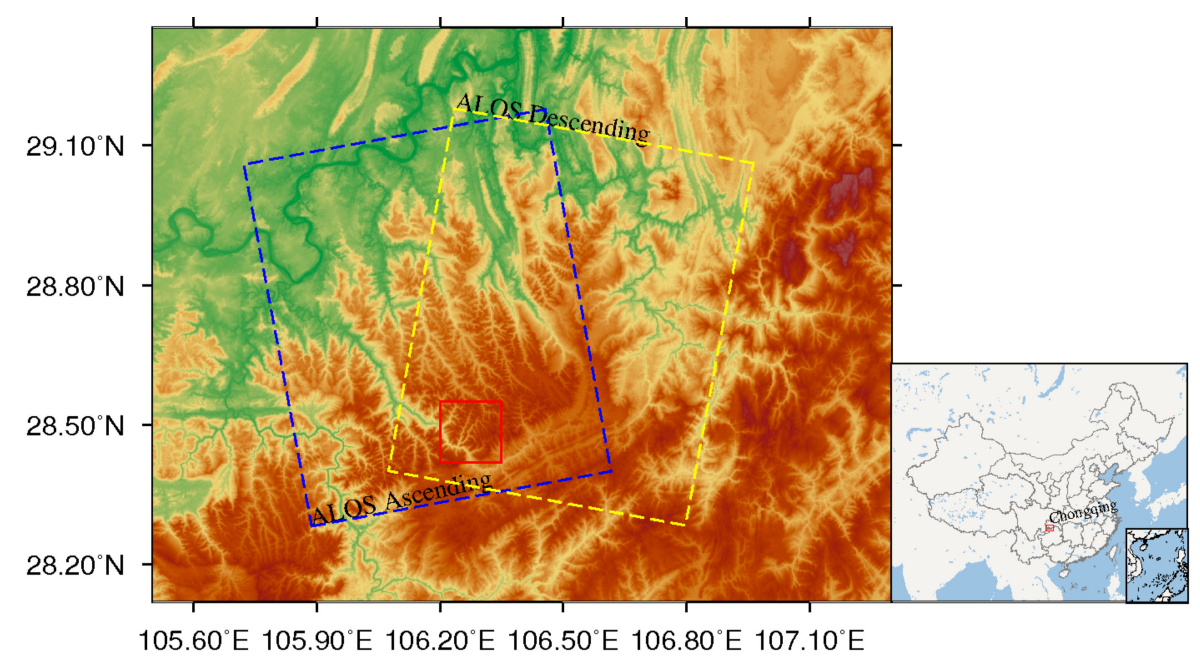

Figure 3. Shaded relief map of Chongqing. The red box indicates the scope of the study area. Blue and yellow dashed boxes indicate the coverage of the ascending and descending ALOS PALSAR datasets, respectively.

\subsection{Results of the P-NG-Derived SGD Mask}

The parameters for the P-NG method were: (1) the iso-azimuth of the ascending and descending LOS of ALOS PALSAR for Chongqing; (2) the incidence angle (near range and far range), the angle the satellite illuminates the Earth's surface; and (3) the local terrain slope and aspect angles from the SRTM DEM data. These parameters were chosen to guarantee worldwide applicability of the proposed method.

Figures 4 and 5 present the comparison of the SGD maps derived by the F-ALS and P-NG methods with respect to the ALOS ascending and descending images, respectively. Since the ALOS ascending and descending data were both recorded at a low incidence angle (around $38.7^{\circ}$ ), most of the areas were dominated by the resolution-enhancing effect. In addition, there were few shadow regions in both images. This indicates that the ALOS data is very suitable for landslide monitoring with InSAR.

To provide more detailed results, a subregion, i.e., the black dashed boxes as shown in Figures 4 and 5, was selected to carry out the comparison. As shown in Figure 4e,f, the areas of layover and shadow obtained by the classical F-ALS method in the ascending data are obviously less than those obtained by the P-NG method that was in possession of the passive distortion areas. By contrast, the simulated layover gained by the P-NG method can highlight the characteristics of the layover on SAR images, i.e., "many-to-one mapping". Besides, it can be found that the range of the foreshortening areas (i.e., " $\mathrm{F}$ ") in Figure $4 \mathrm{f}$ is significantly reduced compared with those in Figure 4e, where the reduced areas are occupied by passive distortion (i.e., "PL" and "PS"). Figure 5 shows the comparison of the SDG maps derived by the F-ALS and P-NG methods in the ALOS descending image, where similar results can be found.

It is not difficult to find that there is a complementary relationship between the ascending and descending data for the simulated SGD in the same region. For instance, many layover distortions in the ascending data correspond to the shadow distortions in the descending data. Furthermore, the regions affected by the SGD in the ascending data are less than those in the descending data, indicating that the ascending data is more suitable for monitoring landslides in the study area.

To further confirm the spatial interaction among the distortions proposed in this paper, we selected several regions which had not been corrected, as shown in Figure 6. The white points represent the passive layover, whereas the black points indicate the passive shadow. It can be noted that whether passive layover or passive shadow, they lie over the partial regions of the foreshortening, respectively. However, beyond that, passive layover interacts with parts of active and passive shadows, and so 
does passive shadow. This phenomenon can be rectified by the P-NG method to obtain the SGD with accurate boundaries.
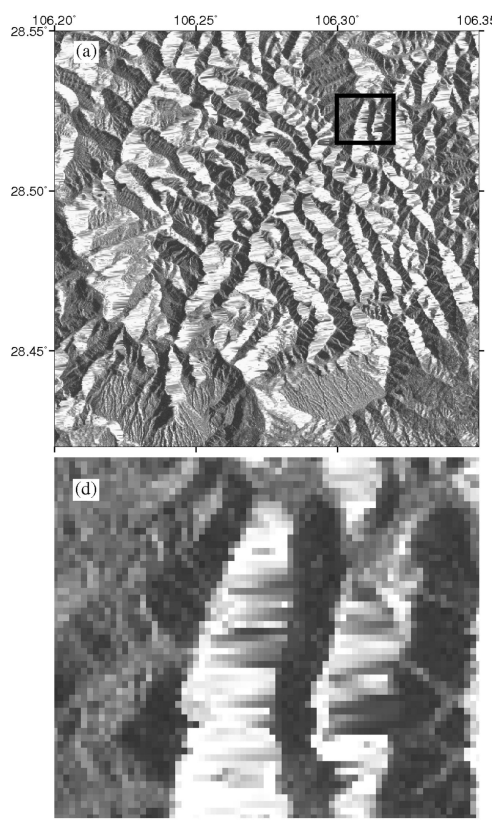
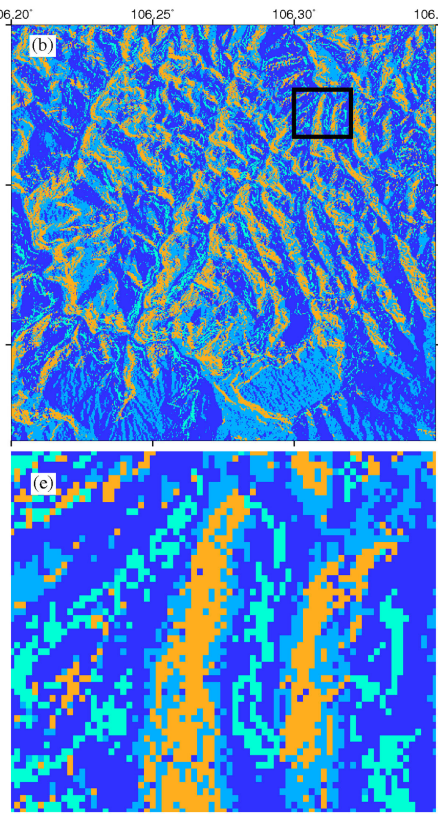
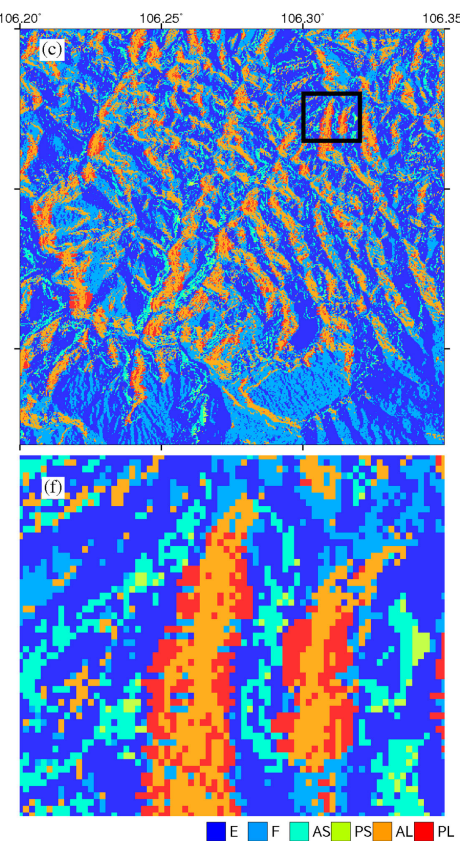

Figure 4. Geometric distortions in the ALOS ascending image. (a,d) display the intensity image; $(\mathbf{b}, \mathbf{e})$ display the SDG map derived by the F-ALS method; (c,f) display the SDG map derived by the P-NG method. E: Resolution-Enhancing; F: Foreshortening; AS: Active Shadow; PS: Passive Shadow; AL: Active Layover; PL: Passive Layover.
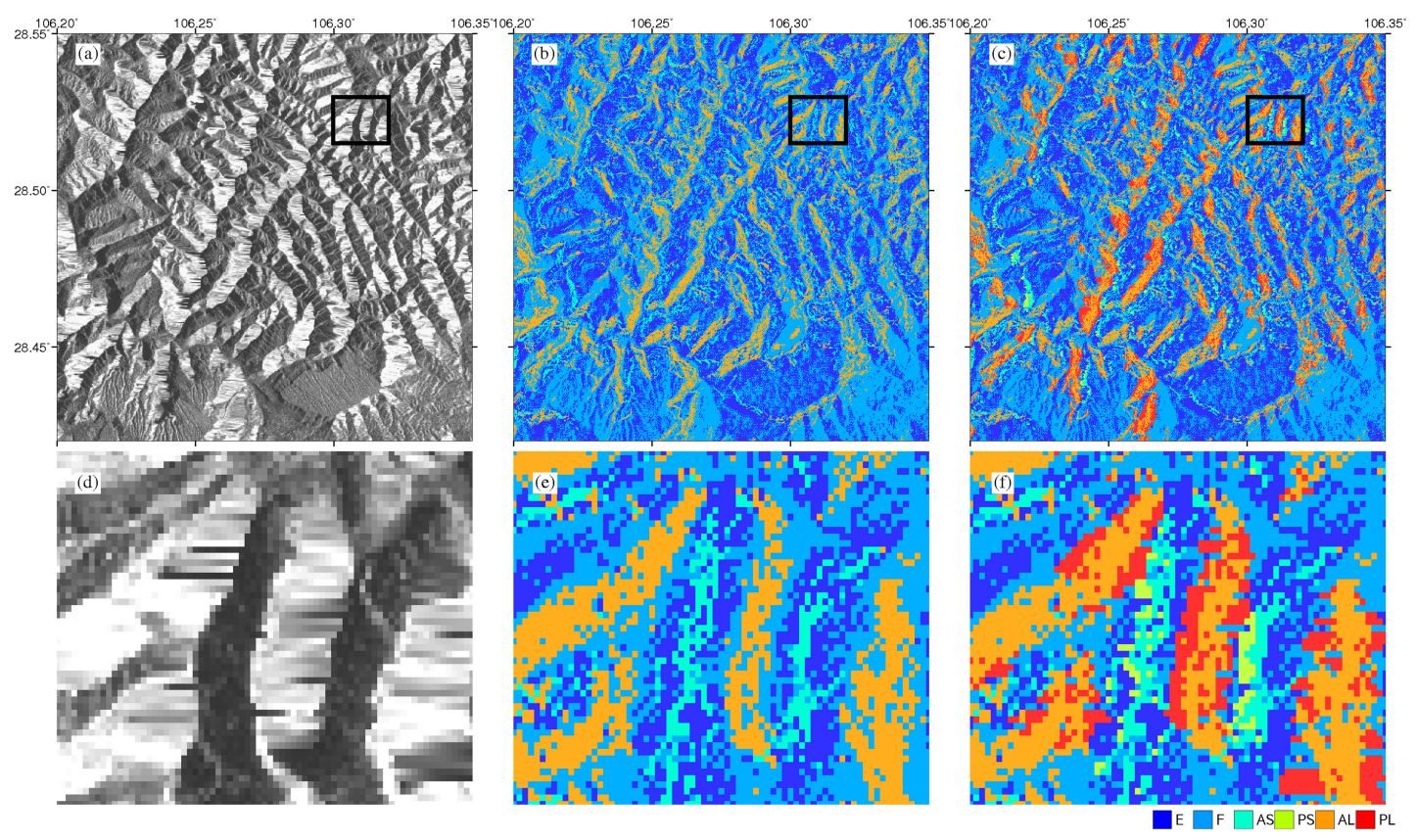

Figure 5. Geometric distortions in the ALOS descending image. (a,d) display the intensity image; $(\mathbf{b}, \mathbf{e})$ display the SDG map derived by the F-ALS method; $(\mathbf{c}, \mathbf{f})$ display the SDG map derived by the P-NG method. E: Resolution-Enhancing; F: Foreshortening; AS: Active Shadow; PS: Passive Shadow; AL: Active Layover; PL: Passive Layover. 

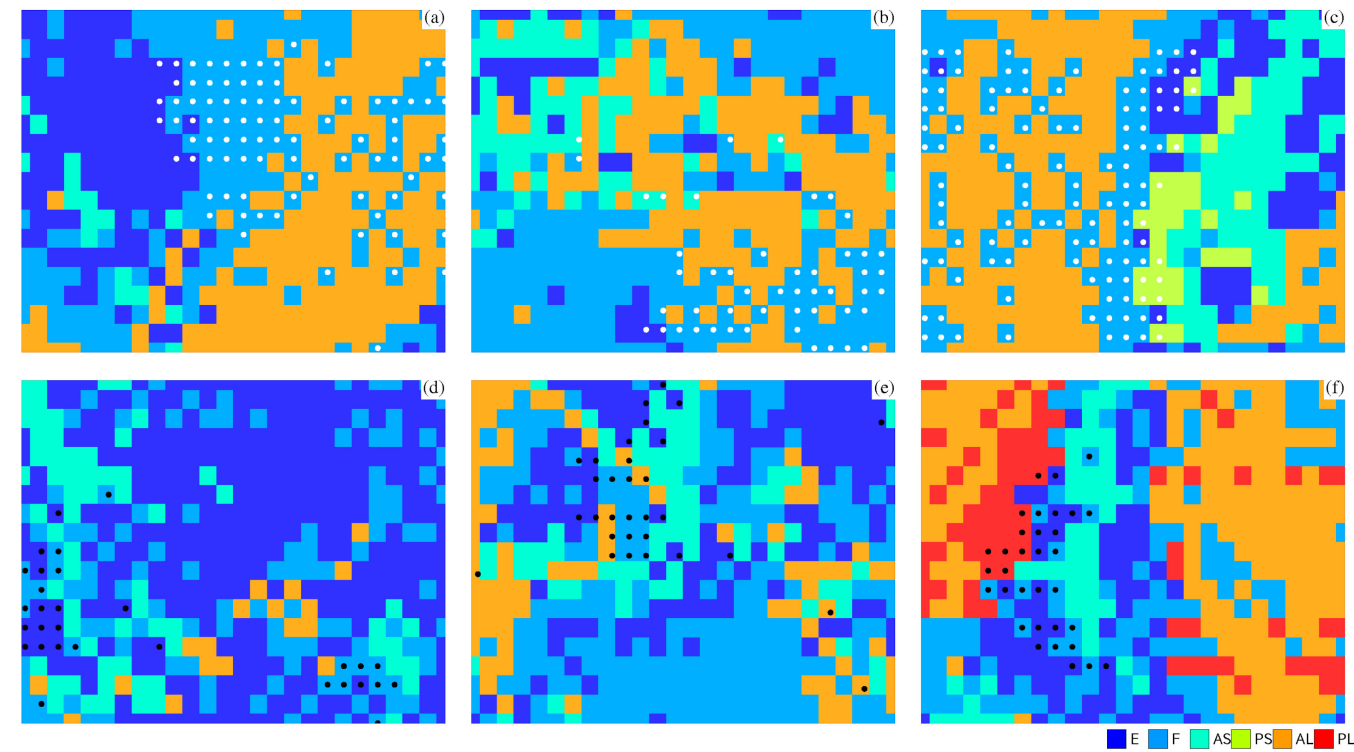

Figure 6. Spatial interactions among the simulated distortions. The white points represent passive layover, while the black points represent passive shadow. (a-c) display the passive layover interacting with the foreshortening ("F"), the active shadow ("AS"), and the passive shadow ("PS"); (d-f) display the passive shadow interacting with the foreshortening ("F"), the active layover ("AL"), and the passive layover ("PL").

\subsection{Comparison Between the SGD and the Interferometric Products}

The interferometric coherence, estimated from two radar recordings within the time interval between the master and slave images, reflects the similarity among echoes [21]. It is used as a metric of the accuracy of measured amplitude and phase. However, low-coherence areas are commonly found in relief mountainous areas affected by the SGD [22]. In addition, both temporal and spatial baselines lead to decorrelation [23-25]. Therefore, the constraints on the temporal and spatial baselines are required for observation of echo correlation to minimize these effects. In this study, since the temporal and spatial baselines are both small and the L-band ALOS PALSAR data has good penetrability, the decorrelation is mainly contributed by these SGD effects.

The coherence maps retrieved from the ALOS ascending and descending pairs are shown in Figure 7. The red and yellow points represent the layover and shadow distortions, respectively, which are superimposed on the coherence, the value of which ranges from 0 to 1.0. As expected, the layover/shadow distortions are basically distributed in the low-coherence regions, which confirms that the areas of the layover/shadow distortion are decorrelated. However, a few layovers have a slightly high coherence where they have tensile distortion. Still, a small part of the layover/shadow points falls in the normal areas. This could be ascribed to the errors of the geo-location of radar scatterers [26]. As shown in Figure 8, the interferometric fringes cannot be well produced in the corresponding layover and shadow regions. This indicates that the SGDs are non-negligible undesirable effects in the InSAR application. 

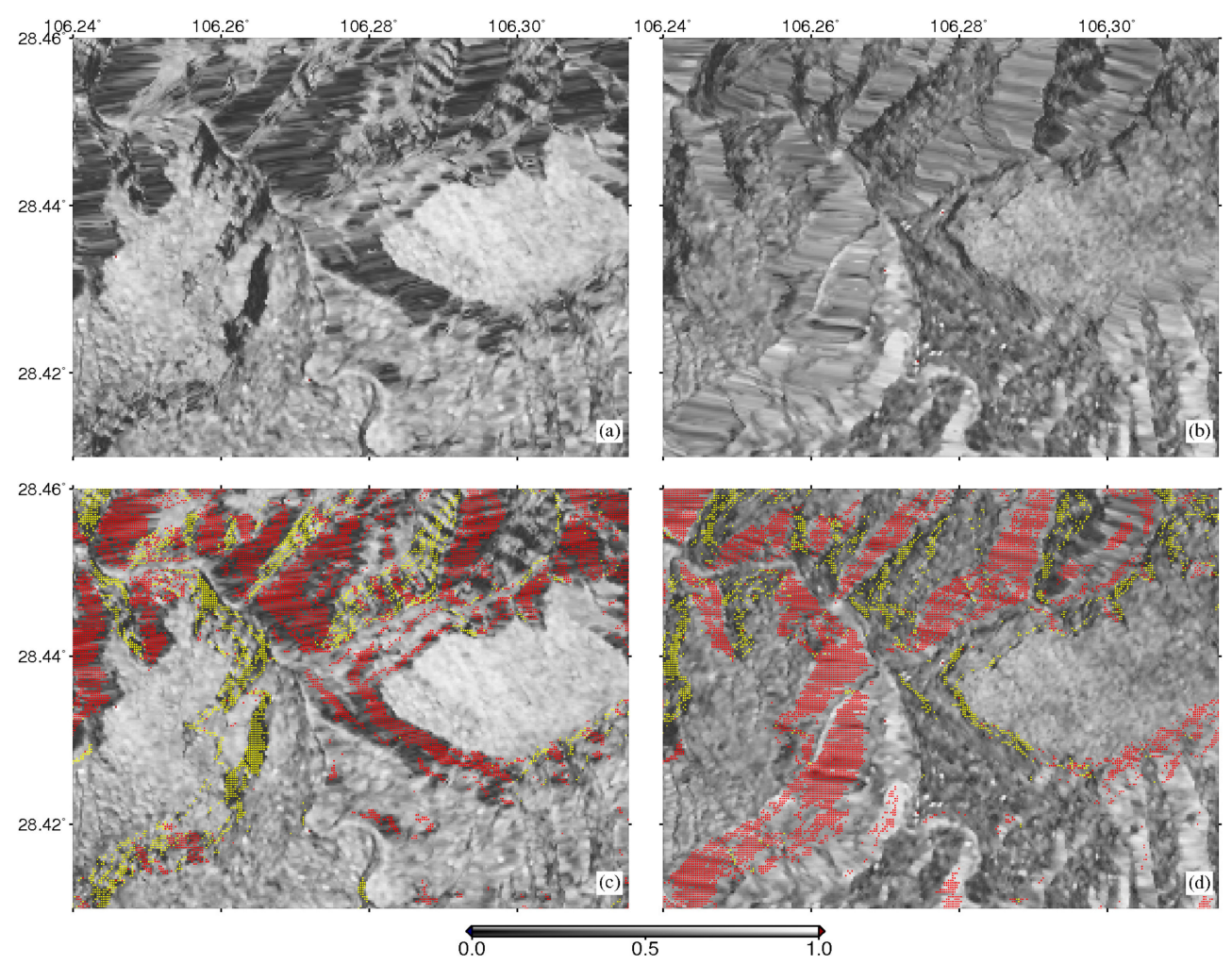

Figure 7. Coherence maps derived from the ALOS ascending data $(\mathbf{a}, \mathbf{c})$ and the ALOS descending data $(\mathbf{b}, \mathbf{d})$; the red points represent the layover and the yellow points represent shadow.
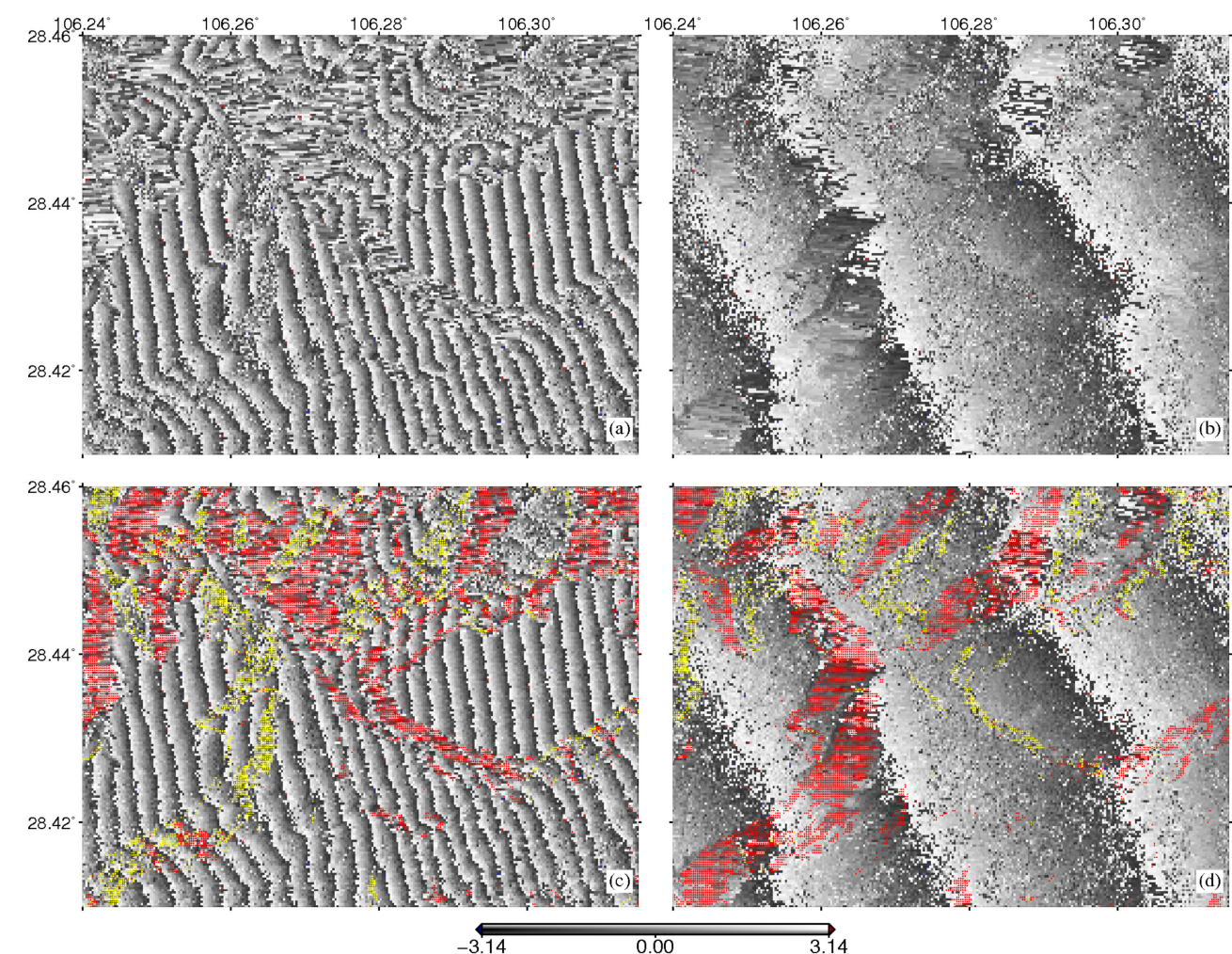

Figure 8. Interferograms derived from the ALOS ascending data $(\mathbf{a}, \mathbf{c})$ and the ALOS descending data $(\mathbf{b}, \mathbf{d})$; the red points represent the layover and the yellow points represent shadow. 


\section{Discussion}

\subsection{Misclassification in the F-ALS-Estimated Distortions}

The P-NG method depends on a pre-classification step obtained with the F-ALS method. The F-ALS method can distinguish areas affected by foreshortening from areas affected by active layover/shadow. Whereas, the method only calculates the relationship between the radar incidence angle and slope angle, and thus cannot consider the passive distortion that might occur in the flat area. This will inevitably lead to misclassification in the F-ALS-estimated results. As shown in Figure 6a,b, the points of the passive layover are found in the F-ALS-estimated foreshortening and active shadow, respectively. Obviously, the overlap area should be redefined as the passive layover according to the interaction of the SGD described in the P-NG method. In addition, it can be found in Figure $6 \mathrm{~d}$,e that the points of the passive shadow lie in the foreshortening and active layover, respectively. The overlap area is a misclassified result of the F-ALS method, but can be redefined as the passive shadow by the P-NG method. Therefore, the misclassification of the F-ALS method will lead to the incorrect coverage of the derived SGD and will limit the application of the SAR interferometry in the mountainous areas, such as landslide monitoring. As mentioned, the P-NG method can correct such misclassifications due to the exploitation of neighbor gradient between active and passive distortions.

\subsection{Effect of Scale Induced by the Input DEM Resolution}

It is worth noting that the above results of the SGD derived by the P-NG method are based on 30-m SRTM DEM data. Since steep and rough terrains are smoothed by lower resolution data, the use of higher-resolution DEM can of course increase the accuracy of simulated SGD by improving the identification of their obtained boundaries and depicting topographic features and complexities.

As discussed in Gelautz et al. [27] and Cigna et al. [13], the resolution of the input DEM is of great significance for the SGD simulation, and the pixel size of the input DEM should be comparable or greater with respect to that of simulated SAR data. However, their observations also confirmed that in the selection of the input DEM there was a trade-off between resolution and cost of the DEM.

To further investigate and quantify the effect of the resolution of the DEM data on the capability of the proposed method in the mapping of the SGD, we conducted a comparison by simulating the layover and shadow with 30-m SRTM DEM as well as 90-m SRTM DEM data.

For facilitating the comparison, the SAR intensity image (geocoded to the geographic coordinates, in Figure 9a) derived from the ALOS descending data was used as the base map. The ALOS descending data, that is, the flight pass of the satellite from the north to the south, operates in a right-looking mode, and the illumination of the test site is from the east to the west. As this image was recorded at an incidence angle of $\sim 38.7^{\circ}$, very strong layover and shadow occurred in the intensity image, in which the layover and shadow regions can be clearly distinguished. They are both classified by visual interpretation, as depicted by the black solid lines in Figure 9, which can be viewed as the "real layover/shadow". 

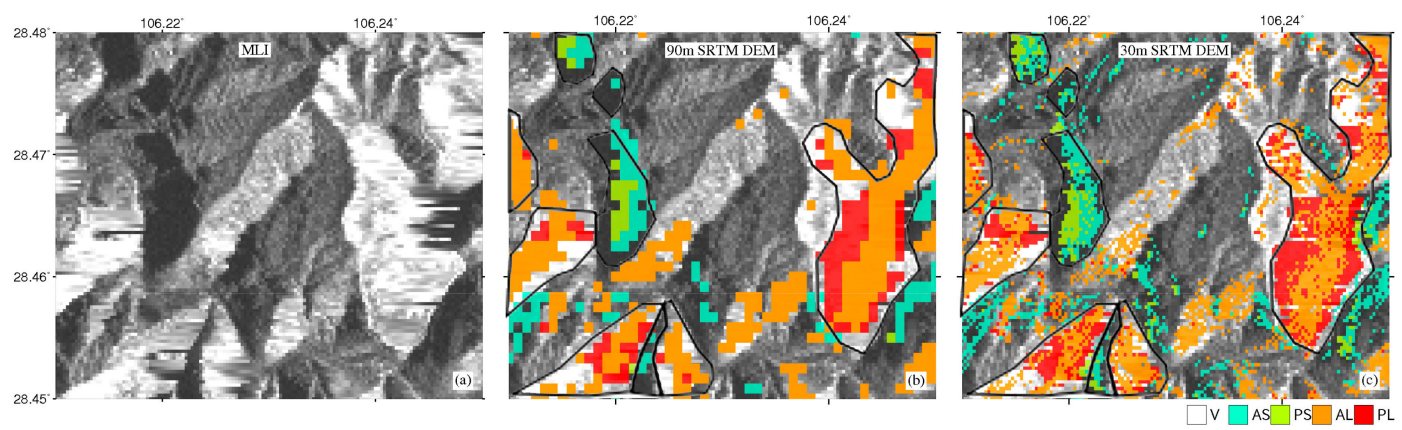

Figure 9. Intensity image of the ALOS descending data (a); the layover/shadow simulated based on 90-m SRTM DEM (b) and 30-m SRTM DEM data (c). V: "real" layover/shadow by visual interpretation; AS: active shadow; PS: passive shadow; AL: active layover; PL: passive layover.

Figure $9 \mathrm{~b}, \mathrm{c}$ shows the results of the layover/shadow simulation using the $90-\mathrm{m}$ and 30-m SRTM DEM data, respectively. In comparison to active layover, it should be noted that the layover distortion with the passive layover is quite compatible with the "real" layover. However, part of the real layover is defined as foreshortening. Similarly, the imperfection can also be found from the results of the simulated shadow. Generally, the areas affected by the layover and shadow derived from 30-m DEM are wider than those from 90-m DEM. Particularly, the details of the layover and shadow identified based on 30-m DEM have been significantly increased compared with those of 90-m DEM. As expected, the higher the resolution of the input data, the more accurate the ranges of the SGD derived by the P-NG method.

Although the performance of the P-NG method highly depends on the resolution of the used DEM, it provides us a good chance to investigate the SGD of a radar image for the study area, which can be used as an analytical reference for InSAR research in this field. The great advantage of the P-NG method is that it can be carried out at a very low cost before radar recording since the underlying DEM data such as SRTM are freely available.

\section{Conclusions}

This paper presented a method called P-NG for simulating the SGD based on the neighbor gradient algorithm. Compared to the classical F-ALS method, the SGD masks derived by the P-NG method are more complete due to the detection of passive distortion. By employing the ascending and descending ALOS PALSAR pairs acquired over the Chongqing area of China, it was found that the areas affected by layover/shadow generated by the P-NG method were dominated by the non-interferometry regions. Therefore, the SGD derived by the P-NG method can also be used as a basis for selecting or optimizing the best collocate of SAR data. In addition, the P-NG method can correct the boundaries among the different effects of the SGD, which greatly improves the accuracy of simulation results. The SGD derived by the P-NG method can greatly improve the use rate of SAR images and enhance the applicability of InSAR in the monitoring of landslides.

Also, the performance of the presented P-NG method depends on the resolution of the used DEM. The realization of a high-resolution Global Elevation Digital Map will be an opportunity for the P-NG method, and the extension of the P-NG method to high-resolution DEM will be our future research topics.

Author Contributions: Q.S. and J.H. conceived and designed the experiments; X.C. performed the experiments; X.C. and Q.S. analyzed the data; X.C. wrote the paper. All authors reviewed and approved the manuscript.

Funding: This research received no external funding.

Acknowledgments: The ALOS PALSAR data were provided by the Japan Aerospace Exploration Agency (JAXA). The research was supported by the National Natural Science Foundation of China (Nos. 41774023, 41674010), the Science and Technology Project of Hunan Province (Nos. 2018JJ3347, 2017RS3001), the Project of Education 
Department of Hunan Province (No. 16K053), and the Land and Resources Research Project of Hunan Province (No. 2017-13). The authors thank three anonymous reviewers for their constructive comments.

Conflicts of Interest: The authors declare no conflict of interest.

\section{References}

1. Kropatsch, W.G.; Strobl, D. The generation of SAR layover and shadow maps from digital elevation models. IEEE Trans. Geosci. Remote Sens. 1990, 28, 98-107. [CrossRef]

2. Hanssen, R. Radar Interferometry: Data Interpretation and Error Analysis; Kluwer Academic Publishers: Dordrecht, The Netherlands, 2001.

3. Eineder, M.; Suchandt, S. Recovering radar shadow to improve interferometric phase unwrapping and DEM Reconstruction. IEEE Trans. Geosci. Remote Sens. 2003, 41, 2959-2962. [CrossRef]

4. Cigna, F.; Bateson, L.; Jordan, C.; Dashwood, C. Nationwide monitoring of geohazards in great britain with InSAR: Feasibility mapping based on ERS-1/2 and ENVISAT imagery. In Proceedings of the IEEE International Geoscience and Remote Sensing Symposium (IGARSS), Melbourne, Australia, 21-26 July 2013; pp. 672-675.

5. Jan, K.; Gianfranco, D.G.; Yrjo, R. Geo-Referencing of Continental-Scale JERS-1 SAR Mosaics Based on Matching Homologous Features with a Digital Elevation Model: Theory and Practice. Remote Sens. 2011, 33, 2413-2433.

6. Deo, R.; Rossi, C.; Eineder, M.; Fritz, T. Fusion of ascending and descending pass raw TanDEM-X DEM. In Proceedings of the 2014 IEEE Geoscience and Remote Sensing Symposium, Quebec City, QC, Canada, 13-18 July 2014; pp. 21-24.

7. Deo, R.; Rossi, C.; Eineder, M.; Fritz, T.; Rao, Y.S. Framework for Fusion of Ascending and Descending Pass TanDEM-X Raw DEMs. IEEE J. STARS 2015, 8, 3347-3355. [CrossRef]

8. Barboux, C.; Delaloye, R.; Strozzi, T.; Collet, C.; Raetzo, H. TSX assessment for slope instabilities monitoring in Alpine periglacial environment (Western Swiss Alps, Switzerland). In Proceedings of the Fringe 2011 Workshop, Frascati, Italy, 19-23 September 2011.

9. Rees, W.G. Simple masks for shadowing and highlighting in SAR images. Int. J. Remote Sens. 2000, 21, 2145-2152. [CrossRef]

10. Notti, D.; Davalillo, J.C.; Herrera, G.; Mora, O. Assessment of the performance of X-band satellite radar data for landslide mapping and monitoring: Upper Tena Valley case study. Nat. Hazards Earth Syst. Sci. 2010, 10, 1865-1875. [CrossRef]

11. Notti, D.; Meisina, C.; Zucca, F.; Colombo, A. Models to predict Persistent Scatterers data distribution and their capacity to register movement along the slope. In Proceedings of the Fringe 2011 Workshop ESRIN, Frascati, Italy, 19-23 September 2011; pp. 17-23.

12. Plank, S.; Singer, J.; Thuro, K.; Minet, C. The suitability of the differential radar interferometry method for deformation monitoring of landslides-a new GIS based evaluation tool. In Proceedings of the 11th IAEG Congress Geologically Active, Auckland, New Zealand, 5-10 September 2010; pp. 2345-2352.

13. Cigna, F.; Bateson, L.B.; Jordan, C.J.; Dashwood, C. Simulating SAR geometric distortions and predicting Persistent Scatterer densities for ERS-1/2 and EVNISAT C-band SAR and InSAR applications: Nationwide feasibility assessment to monitor the landmass of Great Britain with SAR imagery. Remote Sens. Environ. 2014, 152, 441-466. [CrossRef]

14. Colesanti, C.; Wasowski, J. Investigating landslides with space-borne Synthetic Aperture Radar (SAR) interferometry. Eng. Geol. 2006, 88, 173-199. [CrossRef]

15. Sun, Q.; Hu, J.; Zhang, L.; Ding, X.L. Towards slow-moving landslide monitoring by integrating multi-sensor InSAR time series datasets: The Zhouqu case study, China. Remote Sens. 2016, 8, 908. [CrossRef]

16. Liu, W.; Yamazaki, F. Building height detection from high-resolution TerraSAR-X imagery and GIS data. In Proceedings of the 2013 Joint Urban Remote Sensing Event, CD-ROM, Sao Paulo, Brazil, 21-23 April 2013; pp. 33-36.

17. Tapete, D.; Cigna, F.; Donoghue, D.N.M. 'Looting marks' in space-borne SAR imagery: Measuring rates of archaeological looting in Apamea (Syria) with TerraSAR-X Staring Spotlight. Remote Sens. Environ. 2016, 178, 42-58. [CrossRef] 
18. Zhao, L.; Zuo, S.; Deng, D.; Han, Z.; Zhao, B. Development mechanism for the landslide at Xinlu Village, Chongqing, China. Landslides 2018, 15, 2075-2081. [CrossRef]

19. Huang, F.; Chen, L.; Yin, K.; Huang, J.S.; Gui, L. Object-oriented change detection and damage assessment using high-resolution remote sensing images, Tangjiao Landslide, Three Gorges Reservoir, China. Environ. Earth Sci. 2018, 77, 183. [CrossRef]

20. Yang, B.; Yin, K.; Xiao, T.; Chen, L.X.; Du, J. Annual variation of landslide stability under the effect of water level fluctuation and rainfall in the Three Gorges Reservoir, China. Environ. Earth Sci. 2017, 76, 564. [CrossRef]

21. Foster, M.R.; Guinzy, N.J. The coefficient of coherence: Its estimation and use in geophysical data processing. Geophysics 1967, 32, 602-616. [CrossRef]

22. Du, Y.N.; Feng, G.C.; Peng, X.; Li, Z.W. Subsidence Evolution of the Leizhou Peninsula, China, Based on InSAR Observation from 1992 to 2010. Appl. Sci. 2017, 7, 466. [CrossRef]

23. Zebker, H.A.; Villasenor, J. Decorrelation in interferometric radar echoes. IEEE Trans. Geosci. Remote Sens. 1992, 30, 950-959. [CrossRef]

24. Zhang, L.; Sun, Q.; Hu, J. Potential of TCPInSAR in Monitoring Linear Infrastructure with a Small Dataset of SAR Images: Application of the Donghai Bridge, China. Appl. Sci. 2018, 8, 425. [CrossRef]

25. Pege, A.; Calo, F. A Review of Interferometric Synthetic Aperture RADAR (InSAR) Multi-Track Approaches for the Retrieval of Earth's Surface Displacements. Appl. Sci. 2017, 7, 1264.

26. Dheenathayalan, P.; Small, D.; Schubert, A.; Hanssen, R.F. High-precision positioning of radar scatterers. J. Geod. 2016, 90, 403-422. [CrossRef]

27. Gelautz, M.; Frick, H.; Raggam, J.; Burgstaller, J.; Leberl, F. SAR image simulation and analysis of alpine terrain. ISPRS J. Photogr. Remote Sens. 1998, 53, 17-38. [CrossRef]

(C) 2018 by the authors. Licensee MDPI, Basel, Switzerland. This article is an open access article distributed under the terms and conditions of the Creative Commons Attribution (CC BY) license (http:/ / creativecommons.org/licenses/by/4.0/). 